\title{
A rare case of human immunodeficiency virus associated bilateral facial nerve palsy in North India
}

\section{Sanjay Gupta, Jitendra Kumar}

Department of Internal Medicine, Max Super Speciality Hospital, Malsi, Dehradun,

Uttarakhand, India

\section{Address for the Correspondence:} Dr. Sanjay Gupta, Internal Medicine, Max Super Speciality Hospital, Malsi, Dehradun, Uttarakhand, India. E-mail: drsanjay1974@gmail.com

\begin{tabular}{|l|}
\hline Access this article online \\
\hline Website: www.ijmedph.org \\
\hline DOI: $10.4103 / 2230-8598.144133$ \\
\hline Quick response code: \\
\hline
\end{tabular}

Human immunodeficiency virus (HIV) cases are on the increase in India and worldwide, so are its various complications. Neurological complications are important causes of morbidity and mortality in patients with HIV infection. They can occur at any stage of the disease and can affect any level of the central or peripheral nervous systems. In the literature, several cases of HIV-associated facial paralysis have been reported; however, bilateral facial palsy is rarely reported.

Key words: Central nervous system, facial nerve palsy, human immunodeficiency virus

\section{INTRODUCTION}

It is estimated that $40-90 \%$ of patients with primary human immunodeficiency virus (HIV)- 1 infection experience an acute retroviral syndrome. Patients typically present with fever, myalgia, headache, rash, and lymphadenopathy, and the illness can easily be mistaken for mononucleosis, influenza, or other acute illness. ${ }^{[1,2]}$ While unilateral peripheral facial palsy $(\mathrm{PFP})$ is a common disorder with an incidence between 12 and 25 cases/year for each 10,000 habitants, simultaneous bilateral facial palsy is a rare disease, which correspond $<2 \%$ of the facial palsy cases. ${ }^{[1]}$ Its incidence is of one case in 5 millions of people a year. ${ }^{[2]}$ Its occurrence is from $7 \%$ to $12 \%$ of the cases of unilateral PFP, being more common in the opposite side, while the occurrence of bilateral PFP is very rare. ${ }^{[3,4]}$ Occasionally, neurologic complications - including aseptic meningitis, encephalopathy, neuropathy, myelopathy, and brachial neuritis - develop in association with primary HIV-1 infection. Except for encephalopathy, which occurs simultaneously during the acute phase of HIV-1 infection, the other manifestations tend to be observed $\sim 3$ weeks after the onset of the primary illness. ${ }^{[5]}$ Bell palsy has been reported during these episodes and has also been observed in patients who are already known to be infected with HIV-1. ${ }^{4,5]}$ However, facial diplegia experienced at the time of seroconversion is much more infrequent, and was first reported by Wechsler and Ho in 1989. ${ }^{[6]}$

Patients with HIV positive often presents otologic and neurotological manifestations, what means that facial palsy can occur in any stage of the disease, inclusively as first manifestations. ${ }^{[5]}$ Bell's palsy is responsible for the majority of the cases, usually occurring in nonsymptomatic patients. ${ }^{[7}$ We report a rare case of bilateral facial nerve palsy (FNP) associated with HIV-1 infection.

\section{CASE REPORT}

A 32-year-old heterosexual man [Figure 1] who was admitted to the hospital because of fever and confusion and bilateral ptosis.

His past medical history was unremarkable. Fifteen days before admission to the hospital he experienced fever, arthromyalgias, frontal headache, and a maculopapular rash. Treatment with cefuroxime was initiated, without improvement of symptoms. An examination of the patient at admission revealed a maculopapular rash with plantar involvement. The findings of the rest of the physical examination, which included a neurologic examination, were unremarkable. Blood biochemistry results, red blood cell count, and platelet count were within the normal limits. The patient's white blood cell (WBC) count was 10_109 cells/L, with 50\% neutrophils, 36\% lymphocytes, and 13\% monocytes. Blood cultures 
and serological examinations for infection with cytomegalovirus, the hepatitis viruses, Epstein-Barr virus (EBV), Toxoplasma gondii, Brucella species, and syphilis were all negative. However, ELISA and Western blot analyses were positive for HIV-1 infection.

Analyses of cerebrospinal fluid (CSF) samples revealed a glucose content of $2.1 \mathrm{mmol} / \mathrm{L}$ (in blood samples, glucose content was $5 \mathrm{mmol} / \mathrm{L}$ ), a protein content of $1.77 \mathrm{~g} / \mathrm{L}$, and 31 lymphocytes/ $\mathrm{mm}^{3}$. A Gram-stained smear and culture of CSF for bacteria and mycobacteria were also negative. The CD4+ cell count was 825 cells/ $\mathrm{mm}^{3}$ (25\%), and the CD8+ cell count was 1683 cells $/ \mathrm{mm}^{3}$ (51\%). Twenty-four hour after admission, the patient developed bilateral facial palsy. No viral load measurement was available at that time. The patient's condition steadily improved and experienced complete resolution of facial palsy after 3 months.

Patient 2 was a 34 year old heterosexual man with a history of hepatitis $\mathrm{B}$ and epilepsy who was admitted to the hospital because of fever and odynophagia that started 10 days earlier. A nonpruritic maculopapular rash appeared 4 days before hospital admission, and on the day of admission he had the headache, nausea, vomiting, and generalized seizures. An examination of the patient at admission revealed that he was febrile (temperature $38^{\circ} \mathrm{C}$ ) and had cervical, axillary, and groin lymphadenopathies, a generalized rash, involving the palms and soles, and hepatosplenomegaly. The patient's WBC count was 9 _109 cells/L (50\% neutrophils, 37\% lymphocytes, and 8\% bands), and he had an aspartate transaminase level of $204 \mathrm{U} / \mathrm{L}$ (normallevel, !37 U/L), an alanine transaminase level of $206 \mathrm{U} / \mathrm{L}$ (normal level, !41 U/L), lactate dehydrogenase level of $3000 \mathrm{U} / \mathrm{L}$ (normal level, 240-480 U/L), a g-glutamyl transpeptidase level of $489 \mathrm{U} / \mathrm{L}$ (normal level, !54 U/L), and an alkaline phosphatase level of $653 \mathrm{U} / \mathrm{L}$ (normal level, 40-130 U/L). A CSF sample analysis revealed a glucose content of $2.8 \mathrm{mmol} / \mathrm{L}$ (glucosecontent in the plasma sample, $6.0 \mathrm{mmol} / \mathrm{L}$ ), a protein content of $2.29 \mathrm{~g} / \mathrm{L}$, an adenosine deaminase level of $10.5 \mathrm{U} / \mathrm{L}$, and 34 lymphocytes $/ \mathrm{mm}^{3}$ (90\% CD8+ lymphocytes). A Gram-stained smear, culture, and polymerase chain reaction of blood samples for herpes simplex viruses 1 and 2 and enterovirus were all negative. Two-blood sample cultures also had negative results. Serological examinations for cytomegalovirus, EBV, hepatitis viruses A, C, and D, human herpesvirus 6, Toxoplasma species, typhoid fever, and HIV-1, which were requested on admission, all had negative findings. Computed tomography (CT) of the head did not reveal any abnormality. Magnetic resonance imaging (MRI) brain [Figures 2 and 3] showed an abnormal bilateral enhancement of the proximal intracanalicular segments. Three days after hospital admission, patient 2 developed a bilateral PFP. A new serological examination had positive findings for HIV-1 infection. The CD4+ cell count was 586 cells $/ \mathrm{mm}^{3}(8 \%)$ and the $\mathrm{CD}^{+}$cell count was 6011 cells $/ \mathrm{mm}^{3}$ (82\%). Serological examinations for Borrelia species, Brucella species, hepatitis E virus, and adenovirus all had negative findings. The HIV-1 load was (7.2 log10 17_106) copies/ $\mathrm{ml}$. The patient's condition steadily improved over the next 3 months, until the findings of a physical examination were normal except for a moderate bilateral facial palsy that completely resolved after 6 months. He was treated with zidovudine, nevirapine and efavirenz.

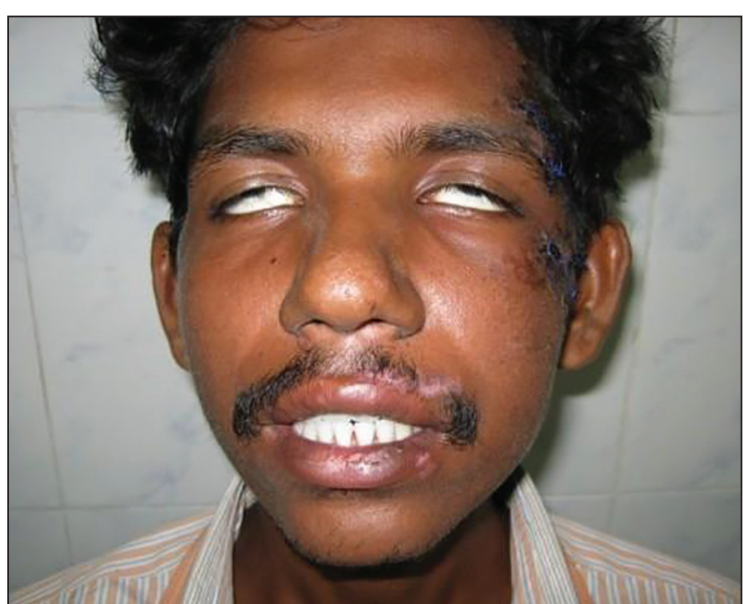

Figure 1: Bilateral facial nerve palsy

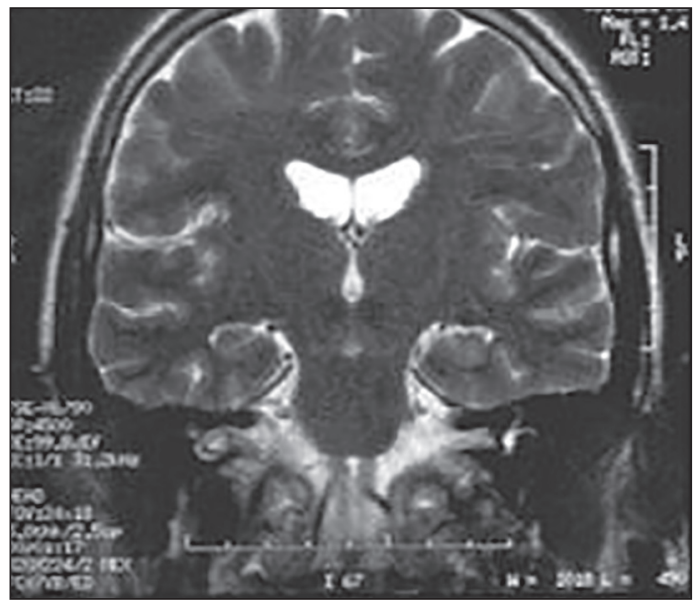

Figure 2: Enhancement of the facial nerve in magnetic resonance imaging

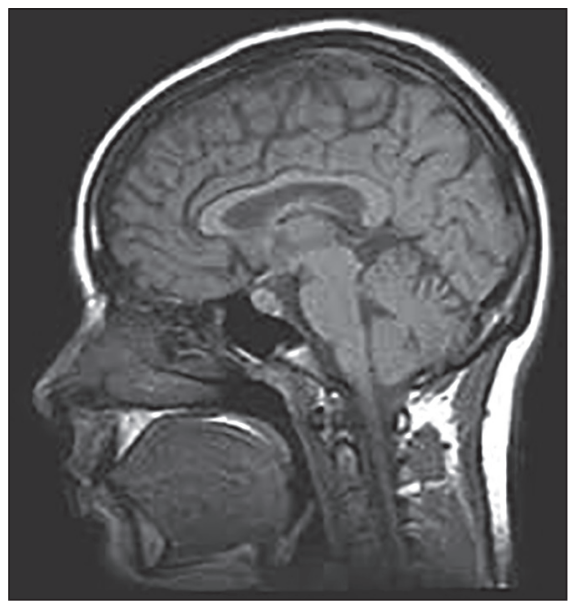

Figure 3: Sagittal section in magnetic resonance imaging

\section{DISCUSSION}

The association of bilateral infranuclear palsy associated with HIV 1 infection was first reported from central Africa in 1985..$^{[8]}$ Bilateral FNP is a rare condition and hence presents a diagnostic 
challenge. Unlike the unilateral presentation, it is seldom secondary to Bell's palsy. The majority of patients with bilateral facial palsy have Guillain-Barre Syndrome (GBS), multiple idiopathic cranial neuropathies, Lyme disease, sarcoidosis, meningitis (neoplastic or infectious), brain stem encephalitis, benign intracranial hypertension, leukemia, Melkersson-Rosenthal syndrome (a rare neurological disorder characterized by facial palsy, granulomatous cheilitis, and fissured tongue), diabetes mellitus, HIV infection, syphilis, infectious mononucleosis, malformations as Mobius syndrome, vasculitis, or bilateral neurofibromas. The possibility of intrapontine and prepontine tumor should also be considered. ${ }^{[9,10]}$ The most common infectious cause of bilateral FNP is Lyme disease, caused by spirochete Borrelia burgdorferi, whose carrier is a common tick. Bilateral FNP can be seen in about 30-35\% of patients with Lyme disease. The pathogenic mechanisms of bilateral Bell palsy in patients who have acute HIV-1 infection are not completely understood. Among the operating mechanisms, direct nerve lesion caused by HIV-1 or an immunologically mediated inflammatory polyradiculopathy, similar to a regional GBS, have been proposed..$^{[1]}$

Diagnosis is serologic, and $\operatorname{IgM}$ antibodies increase in the $2^{\text {nd }}$ week and tend to decrease with treatment, while $\operatorname{IgG}$ antibodies appear late with reaching its peak in the $2^{\text {nd }}$ or $3^{\text {rd }}$ month, and it can indefinitely remain positive. ${ }^{[12,13]}$ GBS is an inflammatory postinfectious polyradiculoneuritis of uncertain etiology. Bilateral FNP can occur in up to $50 \%$ of the fatal cases. The diagnosis is made on clinical findings of peripheral areflexia, and lumbar puncture shows liquoric dissociation: Elevated protein in the absence of raised cell number. ${ }^{[3]}$ Traumatic skull fractures and cerebello-pontine angle tumors were excluded by CT and MRI of the brain. Sarcoidosis was excluded as there was no hilar adenopathy reported on chest X-ray. Diabetes has been noted to be present in $28.4 \%$ of patients with bilateral FNP and can be explained by the fact that diabetic patients are more prone to nerve degeneration. Normal blood sugar levels excluded diabetes as a cause of bilateral FNP in our patient. Approximately, 40\% of the EBV - infection-associated FNP cases are bilateral. ${ }^{[14]}$ There has been a report of brain MRI finding in bilateral Bell's palsy, which showed an abnormal bilateral enhancement of the proximal intracanalicular segments, but no facial nerve swelling. ${ }^{[15]}$

Literature review confirmed that PFP can occur at any stage of HIV infection, however, it is more likely to be present in healthy HIV carriers than in patients with AIDS. ${ }^{[1]}$ Though there have been several reports of facial palsy as a late complication of infection, this neuropathy occurs mainly in the early stages of virus infection, as in our patient. Thus, peripheral palsy can be divided into two types according to etiology; an early stage-related palsy and a late stage-related palsy. ${ }^{[17]}$ In Stages I and II of HIV infection, patients may develop either Bell's palsy or GBS. In Stages III and IV, as cellular immunity begins to decline, herpes zoster-related facial palsy, facial nerve involvement secondary to meningeal lymphomatosis, and PFP, as a manifestation of various chronic peripheral neuropathy, may also occur. ${ }^{[8]}$
Kohler et al. ${ }^{[18]}$ reported seven cases of isolated PFP, with one being a bilateral palsy. It is apparent that there is an increase in facial palsy incidence proportionate to the increase of HIV infection. Two patients with bilateral Bell palsy associated with acute HIV-1 infection have been described in the literature. ${ }^{[19,20]}$ Since facial palsy is considered an initial clinical presentation of HIV infection, it can aid in early diagnosis. High-dose acyclovir may be beneficial in HIV-1-infected patients who have an idiopathic facial palsy, although this has not been systematically studied. Although the disability that is caused by bilateral facial paralysis is dramatically more severe than that which is caused by unilateral paralysis, complete recovery is more commonly reported than incomplete recovery. Of the cases we reviewed, only one patient experienced incomplete recovery. Recovery in bilateral Bell palsy is similar to that in a unilateral palsy, although one side of the face may recover before the other. ${ }^{[21]}$ As early treatment and prevention of HIV dissemination are recommended, it is important to recognize the first signs of HIV infection; and it should be considered as a possible etiology of facial palsy, especially in young age groups.

These cases were started very early on intravenous antiviral drugs and dexamethasone which could have been the reason for their early recovery.

\section{REFERENCES}

1. Colson AE, Sax PE. Primary HIV-1 infection: Pathogenesis, epidemiology and clinical manifestations. UpToDate 2006;311-13.

2. Kassutto S, Rosenberg ES. Primary HIV type 1 infection. Clin Infect Dis 2004 15;38:1447-53.

3. Keane JR. Bilateral seventh nerve palsy: Analysis of 43 cases and review of the literature. Neurology 1994;44:1198-202.

4. Teller DC, Murphy TP. Bilateral facial paralysis: A case presentation and literature review. J Otolaryngol 1992;21:44-7.

5. Denning DW. The neurological features of acute HIV infection. Biomed Pharmacother 1988;42:11-4.

6. Wechsler AF, Ho DD. Bilateral Bell's palsy at the time of HIV seroconversion. Neurology 1989;39:747-8.

7. Casanova-Sotolongo P, Casanova-Carrillo P. Asociación de paralysis facial periférica en pacientes con infección por el virus de la inmunodeficiencia humana. Rev Neurol 2001;32:327-30.

8. Bélec L, Gherardi R, Georges AJ, Schüller E, Vuillecard E, Di Costanzo B, et al. Peripheral facial paralysis and HIV infection: Report of four African cases and review of the literature. J Neurol 1989;236:411-4.

9. May M, Klein SR. Differential diagnosis of facial nerve palsy. Otolaryngol Clin North Am 1991;24:613-45.

10. Serrano P, Hernández N, Arroyo JA, de Llobet JM, Domingo P. Bilateral Bell palsy and acute HIV type 1 infection: Report of 2 cases and review. Clin Infect Dis 2007;44:e57-61.

11. Mateos Romero L, Molinillo López J, Sánchez Román J, Ocaña Medina C. Bilateral facial paralysis as initial symptom in HIV infection. Med Clin (Barc) 2002;118:558-9.

12. Fortes FS, Bento RF, Tsuji RK, et al. Bilateral peripheral facial palsy in HIV patient. Arch Otorhinolaryngol 2005;9:229-33.

13. Pirana S, Bento RF,Bogar P, et al. Paralasia facial e surdez subita bilateral na doenca de Lyme. Rev Bras Otorrinolaringol 1996;62:500-2.

14. Terada K, Niizuma T, Kosaka Y, Inoue M, Ogita S, Kataoka N. Bilateral facial nerve palsy associated with Epstein-Barr virus infection with a review of the literature. Scand J Infect Dis 2004;36:75-7.

15. Shaikh ZA, Bakshi R, Wasay M, Dai A, Gosy E. Magnetic resonance imaging findings in bilateral Bell's palsy. J Neuroimaging 2000;10:223-5.

16. Belec L, Georges AJ, Vuillecard E, Galin M, Martin PM. Peripheral facial paralysis indicating HIV infection. Lancet 1988;2:1421-2. 
17. Sasaki Md, Leite PG, Leite AG, de Almeida SM. Bilateral peripheral facial palsy secondary to lymphoma in a patient with HIVIAIDS: A case report and literature review. Braz J Infect Dis 2002;6:50-4.

18. Kohler A, Burkhard P, Magistris MR, Chofflon M. Isolated peripheral facial paralysis and HIV infection: 7 cases. Rev Neurol (Paris) 1995;151:332-7.

19. Kim MS, Yoon HJ, Kim HJ, Nam JS, Choi SH, Kim JM, et al. Bilateral peripheral facial palsy in a patient with Human Immunodeficiency Virus (HIV) infection. Yonsei Med J 2006;47:745-7.

20. Alcaraz García SF, Muñoz Málaga A, Anglada Pintado JC,
Girón Ubeda JM. Isaolated facial diplegia associated with acute HIV infection. Rev Clin Esp 2003;203:217.

21. Rontal E, Sigel ME. Bilateral facial paralysis. Laryngoscope 1972;82:607-16.

How to cite this article: Gupta S, Kumar J. A rare case of human immunodeficiency virus associated bilateral facial nerve palsy in North India. Int J Med Public Health 2014;4:507-10.

Source of Support: Nil, Conflict of Interest: None declared. 